\title{
L'économie verte, avatar du développement durable
}

La Conférence des Nations unies pour le développement durable, Rio +20 , se tiendra en juin 2012 autour de deux thèmes : 1 'économie verte dans le cadre du développement durable et de l'élimination de la pauvreté ; le cadre institutionnel du développement durable. Si la gouvernance de l'environnement se présente toujours comme un test pour l'architecture institutionnelle des organisations des Nations unies, le thème de l'économie verte marque un rééquilibrage entre les trois "piliers" du développement durable, une nouvelle étape dans les relations de l'économie avec l'écologie et les sociétés.

Mettre l'économie verte au centre des débats, c'est prendre acte du retard de l'économie dans la mise en œuvre du développement durable, c'est inciter les décideurs économiques à modifier leurs usages des ressources. Ce n'est pas par négligence qu'aucune définition de l'économie verte n'est donnée dans le document préparatoire, «Le futur que nous voulons », tant sont vifs les débats en cours dans la société et dans le monde scientifique sur la tendance à confier aux mécanismes de marché la régulation des « biens et services » de la nature.

À l'expectative d'un nouveau cycle vertueux de croissance du capitalisme fondé sur l'écologisation de toute l'économie, s'oppose la dénonciation d'une dépendance technologique et financière accrue vis-à-vis des pays riches. L'économie verte masquerait un protectionnisme vert sous prétexte d'adoption de nouvelles normes environnementales, la conditionnalité de l'aide et l'aggravation des inégalités.

Pourquoi et comment cette économie verte s'est-elle imposée ? Depuis 2008, les États-Unis et les États européens n'en finissent pas de répondre à une crise due à la libéralisation et à la financiarisation de l'économie.

On trouve d'une part la nostalgie des trente glorieuses, où l'État a mené une politique de grands travaux, de protection sociale et de planification d'inspiration keynésienne, sur le modèle du New Deal qui a suivi la crise de 1929. D'autre part, le paradigme technico-économique vert, qui répond à l'idée d'écoefficience, apparaît désormais comme un nouvel horizon du capitalisme. Produire plus avec moins de ressources naturelles et moins de pollutions contribuerait à une meilleure qualité de vie. La prise en compte des changements climatiques a poussé à la recherche et à l'adoption de technologies à basse consommation de carbone, que ce soit dans le domaine du bâtiment, des matériaux, de l'aménagement urbain, des transports, etc. À la modestie de ces réponses technologiques par rapport aux objectifs globaux, répond la fragilité du pilier social du développement durable.

Parallèlement à cette technologie décarbonnée, dite « verte ", s'est développée dans le monde de la conservation une vision toujours plus marchande de la nature. La Convention sur la diversité biologique (CDB) de 1992 a consacré le partage juste et équitable des avantages tirés de l'exploitation des ressources génétiques, ouvrant ainsi sur des controverses autour de la brevetabilité du vivant et de marchés potentiels entre acheteurs et fournisseurs de ressources génétiques. La Convention-cadre des Nations unies sur les changements climatiques a, quant à elle, favorisé la création d'un marché des émissions de gaz à effet de serre. Ce marché s'est imposé lors de la conférence de Durban comme seul engagement obligatoire des pays industrialisés et doit être étendu à tous les pays à l'horizon 2020.

Le domaine des représentations scientifiques a également été bouleversé. La CDB a ouvert la voie en prônant l'échelle des écosystèmes pour la régulation de la biodiversité. Puis les écosystèmes ont été présentés comme fournisseurs de services pour le bien-être de l'homme. La communauté des scientifiques n'étant pas étrangère à celle des opérateurs du développement, les paiements pour services environnementaux sont devenus outils de conservation. Ceux-ci impliquent la définition de droits de propriété, de méthodes d'évaluation monétaire, d'unités de mesure, la création de marchés de droits, de marchés de compensation... Le modèle théorique idéal est alors celui d'un échange régulé par le marché. Que ce service ou ce dommage soit évalué par équivalence monétaire (soutenabilité faible) ou par le coût du maintien des 
fonctionnalités de l'écosystème (soutenabilité forte), une ultime traduction monétaire est calculée.

Pour mesurer les bénéfices et dommages portés à l'environnement, il importe de procéder à l'évaluation des «biens et services » de la biodiversité et du capital naturel (autant de métaphores économiques), de réfléchir sur les limites des indicateurs de richesse comme le PIB (un des attendus de Rio + 20), sur des indicateurs pour une comptabilité verte. S'ensuit une multiplication de rapports sur l'économie de la biodiversité et du développement durable (cf. Salles, J.-M., 2010. Évaluer la biodiversité et les services écosystémiques : pourquoi, comment et avec quels résultats ?, NSS, 18, 4, 414-423) qui définissent des politiques de conservation de la nature au prisme des mécanismes de marché.

L'économie verte est née de cette collusion entre science et politique. Les débats de Rio + 20 en montrent déjà les limites et la nécessité de repenser les modèles proposés. Dans un éditorial précédent («Rio + 20 : le développement durable à la croisée du local et du global ?», NSS, 19, 4, [2011]), Bernard Hubert et Jean-Paul Billaud s'interrogent sur le rôle de la science dans les conventions internationales d'environnement. Partant du constat de la difficulté des scientifiques à répondre aux attentes des décideurs pour fonder des accords globaux, ils plaident pour un universalisme qui repose non pas sur une action politique négociée à l'échelle globale, mais sur un partage de valeurs fondé sur les expériences locales. La diversité des nouveaux rapports entre sciences et sociétés s'observe en effet dans les démarches transdisciplinaires à l'œuvre dans des expériences localisées de gestion des ressources et de vivre-ensemble. Cette imagination du local offre autant de réponses aux enjeux globaux.

$C^{\prime}$ est bien cet exercice de mise en critique, de confrontation de valeurs et d'observation d'expériences locales que poursuit NSS. La gestion des écosystèmes comme les thèmes des conventions ne peuvent être socialement ni localement déconnectés.

On trouvera dans cette livraison de NSS plusieurs textes qui renvoient à une analyse critique de cette collusion. Philippe Méral interroge l'émergence de la notion de service écosystémique, tandis qu'Harold Levrel et al. mettent en perspective plusieurs méthodes d'évaluation économique. On trouvera aussi un dossier consacré à Elinor Ostrom, prix Nobel d'économie, qui analyse les systèmes de gestion associés à des biens communs (commons pools of ressources). Ces systèmes autogouvernés, indépendants des lois de l'État, du salariat ou du marché, peuvent s'avérer plus performants que les systèmes de régulation marchande reposant sur la propriété privée des ressources.

La richesse de ces approches parallèles est la contribution de NSS aux controverses de la conférence Rio +20 .

Catherine Aubertin

Alors que nous validions les épreuves de ce numéro, nous avons appris avec une grande tristesse le décès, le 8 avril dernier, de Jean-Marie Legay, cofondateur de la revue Natures Sciences Sociétés avec Marcel Jollivet et Gérard Mégie. Nous lui rendrons hommage dans un prochain numéro, avec la publication de plusieurs contributions témoignant de son envergure humaine et intellectuelle.

La Rédaction 